\title{
Civilisations
}

Revue internationale d'anthropologie et de sciences

humaines

$39 \mid 1991$

Japon : les enjeux du futur

\section{Industrie du terroir ou industrie mondiale : l'électronique japonaise au-delà des compteurs}

\author{
Jean-Louis Perrault
}

\section{OpenEdition}

\section{Journals}

Édition électronique

URL : http://journals.openedition.org/civilisations/1669

DOI : 10.4000/civilisations. 1669

ISSN : 2032-0442

\section{Éditeur}

Institut de sociologie de l'Université Libre de Bruxelles

\section{Édition imprimée}

Date de publication : 30 octobre 1991

Pagination : 399-444

ISBN : 2-87263-044-9

ISSN : 0009-8140

Référence électronique

Jean-Louis Perrault, «Industrie du terroir ou industrie mondiale : I'électronique japonaise au-delà des compteurs », Civilisations [En ligne], 39 | 1991, mis en ligne le 06 juillet 2009, consulté le 03 mai 2019. URL : http://journals.openedition.org/civilisations/1669; DOI : 10.4000/civilisations.1669 


\title{
INDUSTRIE DU TERROIR OU INDUSTRIE MONDIALE : L'ELECTRONIQUE JAPONAISE AU-DELA DES COMPTEURS
}

\author{
Jean-Louis PERRAULT
}

"La tyrannie industrielle de l'Angleterre sur le monde est la domination de l'industrie sur le monde. L'Angleterre nous domine parce que l'industrie nous domine. Nous ne pouvons nous libérer de l'Angleterre à l'extérieur qu'en nous libérant de l'industrie à l'intérieur. Nous ne pouvons tuer sa domination fondée sur la concurrence qu'en surmontant la concurrence à l'intérieur de nos frontières. L'Angleterre exerce de la puissance sur nous parce que nous avons fait de l'industrie une puissance qui nous domine".

Karl MARX, "Critique de l'Economie Nationale" E.D.I., 1975 (1845), Paris, p. 92.

"Nous savons (...) par expérience que bien des causes s'opposent a la sortie des capitaux. Telles sont : la crainte bien ou mal fondée de voir s'anéantir au dehors un capital dont le propriétaire n'est pas le maître absolu, et la répugnance naturelle qu'éprouve tout homme à quitter sa patrie et ses amis pour aller se confier à un gouvernement étranger (...). Ces sentiments, que je serais fâché de voir affaiblis, décident la plupart des capitalistes à se contenter d'un taux de profit moins élevé dans leur propre pays, plutôt que d'aller chercher dans des pays étrangers un emploi plus lucratif pour leurs fonds".

D. RICARDO, "Des principes de l'Economie Politique et de l'Impôt", Champs, Flammarion, Paris, 1977 (1817), p. 118. 


\section{Introduction}

"L'Etat Nation par son intégration dans l'économie mondiale devient un territoire économique" écrit C.A. Michalet ${ }^{1}$. Dans le cas de l'industrie électronique japonaise, cette assertion se vérifie mal. Fort extravertie si l'on observe le compteur que constituent les exportations - 52,7\% de la production exportée en 1984 dont $68 \%$ dans l'électronique grand public - elle apparaît complètement repliée sur son territoire au regard des importations - $5 \%$ de la demande intérieure en 1985 -. Il y a engagement inductif de l'industrie électronique japonaise - ou du Japon dans l'électronique - si l'on se réfère aux grilles d'analyses proposées par Gérard Lafay².

Nous n'irons pas plus loin dans ce relevé des compteurs que constitue l'analyse des exportations et des importations ${ }^{3}$, car plus qu'une mesure des flux, nous souhaitons observer les tensions qui ont amené l'industrie électronique japonaise à engager un processus de délocalisation et à casser, pour ce faire, son système de production territoriale. Le protectionnisme dont ont bénéficié les industriels japonais est désormais bien connu. Ajouté à la planification incitative du M.I.T.I. et au rôle prospecteur du Keidanren, il a permis le développement de l'industrie électronique japonaise sous l'insémination des techniques occidentales, mais nous devons noter que ces résultats ont été obtenus au moyen d'un ensemble industriel peu ouvert aux implantations étrangères, et très peu transnationalisé lui-même.

Le quasi-contrôle du prix des produits intermédiaires de l'électronique, bien que l'industrie se cantonne au Japon, a conduit à des mesures de rétorsion lorsque certaines productions japonaises ont conquis l'exclusivité dans d'autres pays, soit qu'elles évinçassent d'autres producteurs, soit qu'elles se présentassent avec un système de prix trop puissant. Nous 
allons décrire dans une première section la chronologie des délocalisations japonaises dans les années 1960 et au début des années 1970 . Ce mouvement ne résorbe aucunement les frictions commerciales, bien au contraire, celles-ci s'amplifient vis-à-vis des Etats-Unis notamment. Dans un contexte de déclin de l'industrie manufacturière, les gouvernements acceptent mal la fragilisation de l'indépendance industrielle dans un compartiment technique essentiel pour l'avenir. L'observation des soldes commerciaux souligne la montée de la domination japonaise à la fin des années $1970^{4}$. Les frictions protectionnistes qui en résultent amènent une deuxième vague de délocalisation des firmes japonaises, somme toute également assez timide. Il faut que le taux de change du Yen se réévalue de façon dramatique en 1985 pour que le mouvement s'amplifie. Nous décrirons cette période dans une seconde section.

On peut s'interroger sur les raisons qui ont différencié à ce point les firmes nord-américaines et les firmes japonaises dans leurs stratégies de mondialisation. $\mathrm{Si}$, comme le souligne Marc Humbert, "le phénomène de multinationalisation a en quelque sorte constitué un substitut à une ouverture imparfaite" 5 , on s'explique mal pourquoi l'ampleur des délocalisations japonaises est restée infiniment moindre que celle des firmes nord-américaines. Nous tenterons de comprendre ce décalage dans la troisième section.

\section{Section I. Le terroir dominant}

Jusqu'au milieu des années soixante-dix, la délocalisation des producteurs japonais ne se fait remarquer que dans quelques pays d'Asie qui présentent des avantages de salaires et où les Japonais sont bien accueillis (Corée, Taiwan, Thailande). Dans la période initiale, 1950-1971, la demande intérieure, celle de l'Amérique (pour les textiles et pour l'acier surtout) et celle des pays d'Asie qui ont les moyens d'importer suffisent à 
l'industrie japonaise alors que le Yen est sous-évalué (§ 1). Dans la période qui vient ensuite et qu'on peut étendre jusqu'à 1980, des tensions protectionnistes se révèlent et, avant même la révision des cours, elles obligent certaines firmes japonaises à reconsidérer leur stratégie moniste de mondialisation par l'exportation (\$2).

$\S 1$ - Un repli absolu : 1950-1971

Les premiers cas qui permettent d'entrevoir la délocalisation datent de 1951, année d'établissement de filiales commerciales aux Etats-Unis par les maisons de commerce et quelques entreprises manufacturières. Après cette date, l'I.D.E. ${ }^{6}$ commence à prendre un léger essort suivant deux axes. D'abord celui des pays industrialisés dans lesquels les implantations sont commerciales. En second lieu, celui des pays moins développés attirant les investissements japonais sur les sites de matières premières (Koweit, Indonésie, Malaisie, Salomon), accessoirement sur des lieux de pêche. D'une manière comparable à l'investissement entre 1951 et 1971, l'essentiel de l'effort de délocalisation est venu des secteurs commerciaux et financiers $(47,8 \%)$ ou des industries agroalimentaires (I.A.A.) et du secteur minier $(24,7 \%)$. L'industrie manufacturière n'y a contribué de façon notable que dans les secteurs des textiles, du bois et dérivés et de la sidérurgie $(15,8 \%$ de l'I.D.E. cumulé pendant cette période cf. tableau 1). 
Tableau 1. Géographie du stock d'investissements japonais à l'étranger dans l'industrie manufacturière (millions de dollars)

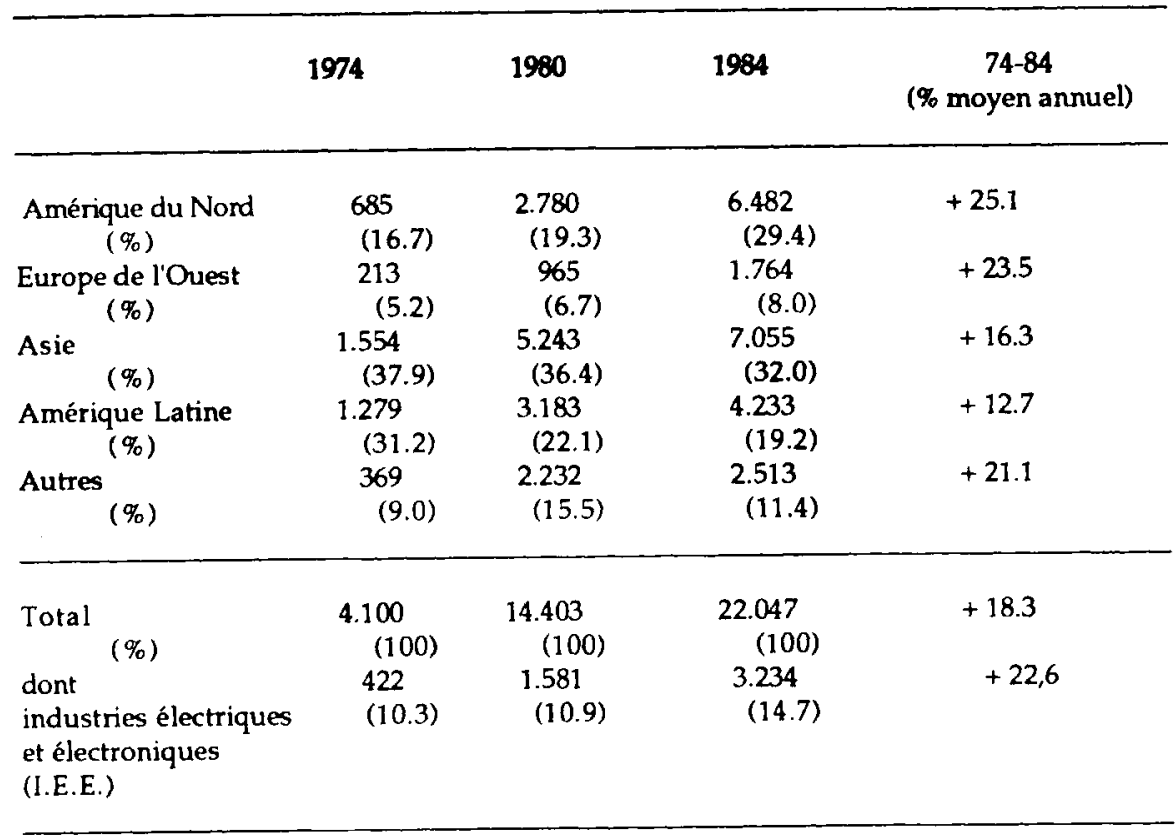

Les autres branches de l'industrie manufacturière ont contribué seulement à $11,7 \%$ de ce dernier. Par contre, l'industrie électronique japonaise, comme celle de la chimie, s'est approvisionnée en techniques étrangères au point que l'on pourrait dire que l'essentiel des technologies de base a été importé. Ainsi en 1954, la Tokyo Tsushin Kogyo (Sony) achète la technologie du transistor des Bell Labs et Nippon Electric Co. celle des circuits intégrés Planar, en 1963.

Alors que les firmes Nord-américaines produisaient 29 millions de transistors et les japonaises 6 millions en 1957, ces dernières en produisirent 180 millions en 1961 et les américaines 190. Or les salaires japonais n'atteignaient alors que le tiers des 
salaires américains. Quelques fabricants américains envisagèrent d'automatiser complètement leur production malgré l'inélasticité que cela entraîne. D'autres se délocalisèrent en fonction des zones de salaires. Fairchild ouvrit la voie, en 1962, en créant une unité de production à Hong-Kong, puis en République de Corée et à Taiwan. La plupart des autres fabricants firent de même, installant une quantité appréciable d'unités d'assemblage en Asie du Sud-Est ${ }^{7}$. Ayant provoqué les premiers mouvements de délocalisation vers les pays à main- d'oeuvre bon marché, les entreprises japonaises contribuèrent malgré tout assez peu à ce mouvement, car elles étaient servies par un taux de change du yen très favorable (360 yens pour un dollar en 1965), et par des tarifs douaniers "éducateurs".

L'ouverture du Japon, réclamée par les Américains en 1960, puis la raréfaction relative de la main-d'oeuvre professionnelle pendant la haute croissance, le changement des méthodes, le déménagement des unités de production à l'intérieur du Japon, poussèrent à la production de masse avec des salaires individuels désormais plus élevés. Cependant, les industriels conservèrent des productions anciennes pour leurs exportations en Asie (économies d'échelle déjà faites). Le rapport des exportations à la production passa de $10 \%$ en 1960 à $30 \%$ dix ans plus tard. Les firmes japonaises augmentèrent l'intensité capitalistique et automatisèrent les procédés qui s'y prêtaient. La relocalisation de leurs unités de production n'était encore que provinciale. Mais une certaine expérience acquise autorisait d'autres essais. L'occasion commença à se présenter lorsque l'éclatement du système monétaire international amena une forte hausse du yen. 
$\$ 2$ - L'amorce des délocalisations : 1972-1980

La formation de capitaux supplémentaires amenée par la dévaluation du dollar fut tout à fait considérable. Peu de temps après, l'inflation de la première crise pétrolière et la nouvelle façon de considérer l'énergie firent envisager plus facilement par les entreprises l'investissement de production outre-mer. Trente-deux milliards de dollars sont investis à l'étranger entre 1971 et 1980 (cf. tableau 1) et répartis équitablement entre les pays industrialisés (14 milliards) et les P.V.D. (17 milliards). Cependant, la structure de cet investissement varie très nettement entre ces derniers, où l'industrie manufacturière est la dépositaire de presque $45 \%$ de l'I.D.E. total et les pays industrialisés pour lesquels l'I.D.E. manufacturier ne représente que $25 \%$. Le secteur minier est encore privilégié avec $40 \%$ des capitaux. En revanche, les industries électriques et électroniques restent marginales au sein de ces flux où elles n'atteignent pas $5 \%$. Ce sont les industries lourdes ou anciennes (chimie, sidérurgie, textiles) qui apparaissent comme les principaux objets du transfert de capital, le tertiaire demeurant un des grands acteurs du mouvement.

Dans l'industrie électronique, des raisons exogènes ont renforcé la tendance. L'une d'elles est la création de zones franches manufacturières en Asie. Ces zones se sont développées après 1970. Elles ont attiré les producteurs japonais de matériels d'électronique grand public qui ont été suivis par une partie de leurs sous-traitants (fabricants de pièces et de composants). Ces derniers ont également défendu leurs parts de marché auprès des constructeurs américains de télévision implantés en Asie du Sud-Est (exemples de G.E., R.C.A., Zenith). Toutefois, en marge de l'occasion offerte par les zones franches, l'exposition au protectionnisme a joué le plus grand rôle dans la motivation des producteurs japonais à mondialiser leur production. L'électronique grand public en 
fournit bien l'illustration (tableau 2).

Tableau 2. La vague de délocalisation vers les P.V.D.

Firme

Pays d'implantation

Année

T.V. Hitachi

Taiwan

$1928^{1}$

T.V. Philips

Taiwan

1956

C. General Instrument

Taiwan

1957

T.V. Matsushita Electric

Taiwan

1962

C. Fairchild

Corée

1966

C. Signetics

Corée

1966

T.V. Motorola

Corée

1967

T.V. R.C.A.

Taiwan

T.V. Philips

C. Electronics Co

Indonésie

1967

Corée

1968

Singapour

1969

C. Hewlett Packard

Corée

1970

C. Microsystems

Thaillande

1970

T.V. Sanyo

Taiwan

1970

T.V. Zenith

Corée

1971

C. Tokyo Electronics

Malaisie

1971

C. Hitachi

Malaisie

1972

C. Hewlett Packard

Malaisie

1972

C. Intel

C. Texas Instruments

Malaisie

1972

C. National Semi Conductor

Malaisie

1972

C. National Semi conductor

Thaillande

1972

1973

C. Signetics

Thaillande

1973

C. Fairchild

Indonésie

1974

T.V. Hitachi

Malaisie

1974

C. Philips

Corée

1974

\section{Source : GERDIC}

C : composants

TV : téléviseurs

1. Il s'agissait alors de radio et de matériel électrique. Taiwan faisait alors partie de l'Empire nippon. 
En 1971, l'U.S. Tariff Commission accuse de dumping les fabricants japonais de téléviseurs, accélérant la vague de délocalisation. En 1976, un tiers des téléviseurs couleur et la moitié des téléviseurs noir et blanc vendus aux Etats-Unis sont importés. Pour limiter ce phénomène, le gouvernement américain impose aux produits venant d'Extrême-Orient l'Orderly Marketing Agreement (O.M.A.), c'est-à-dire des quotas à l'importation aux Etats-Unis. Le premier O.M.A. prend effet vis-à-vis du Japon en 1977 et deux accords supplémentaires sont passés avec Taiwan et la République de Corée du Sud en 1979. Le premier expirera en 1980 et les seconds en juillet 1982; toutefois, ils ne font que suivre le mouvement de délocalisation des firmes japonaises, puisque Sony avait implanté une filiale en Californie dès 1972 et que Matsushita avait racheté Quasar, la filiale Motorola, en 1974. Enfin Sanyo rachète Warwick, le principal fournisseur de la chaîne commerciale Sears, en $1976^{8}$. En revanche, cette législation a provoqué la création d'unités de production des groupes taiwanais Tatung, puis Sampo en Californie et en Géorgie en 1970 et 1981, puis celle du groupe coréen Gold-Star (filiale de Lucky), en Alabama en 1982. Les fabricants nordaméricains, comme Zenith ou R.C.A., subiront également les quotas puisqu'ils exportaient l'ensemble de leur production sous les tarifs 806-3 et 807, depuis Taiwan. De la même façon, tous les téléviseurs importés du Mexique aux Etats-Unis pendant les années soixante-dix ont été produits sous contrat de firmes nord-américaines ${ }^{9}$. A compter de cette période, les flux d'échange de produits de l'E.G.P. ne sont plus lisibles avec les instruments habituels de l'économie internationale.

Dans le cas des composants électroniques et plus précisément des semi-conducteurs, les motifs de délocalisation des firmes Nord-américaines relèvent davantage d'une compétition entre elles et d'une volonté de rendre plus flexibles les capacités de production ${ }^{10}$; mais ces flux sont intenses et 
troublent aussi les résultats qui apparaissent lors du calcul des balances commerciales.

A l'issue de cette seconde période, les entreprises japonaises ont bien amorcé la délocalisation de leurs unités de production. Toutefois, aussi loin que les statistiques disponibles nous permettent de le voir, la segmentation des processus productifs reste timide, comme le montrent les données du tableau 3, faisant ressortir une faible proportion de production à l'étranger. Globalement, les firmes japonaises ont continué à constituer une "base productive ramassée". Evidemment les maisons mères japonaises ont réalisé avec leurs filiales des flux d'échanges importants (tableau 4); les I.E.E. japonaises ont importé 41,9\% de leurs produits depuis les filiales en 1983. Mais les maisons nord-américaines dans les mêmes secteurs ont fait plus (57,8\% en $1982 ; 50,1 \%$ si l'on excepte l'informatique).

Enfin, si on rapporte les flux intra-firmes au commerce extérieur total (pour le Japon ou les Etats-Unis) la concentration territoriale de l'industrie japonaise saute aux yeux $(9,6 \%$ seulement d'exportation vers les filiales à l'étranger, en 1983; 3,1\% d'importation de ces filiales. Pour les firmes américaines, les chiffres sont de 20,4 et $14,6 \%$ ). 
Tableau 3. Extraversion des bases productives : une comparaison (1977)

\begin{tabular}{lcccccc}
\hline & \multicolumn{2}{c}{ C.A. à l'étranger (a)(\%) } & \multicolumn{2}{c}{ Production à l'étranger (b)(\%) } \\
& Japon & Etats-Unis & Europe & Japon Etats-Unis & Europe \\
\hline & 29,7 & 34,5 & 56,4 & 6,7 & 29,2 & 37,5 \\
TOTAL dont & - & 45,9 & 63,4 & 1,6 & 42,2 & 42,5 \\
$\begin{array}{l}\text { Matériel de bureau } \\
\begin{array}{l}\text { Matériel de mesure, } \\
\text { de photographie }\end{array}\end{array}$ & - & 46,4 & - & - & 35,2 & 5,6 \\
$\begin{array}{l}\text { Matéricls électriques } \\
\text { et électroniques }\end{array}$ & 24,4 & 33,4 & 45,9 & 4,3 & 21,2 & 27,9 \\
\hline
\end{tabular}

(a) ventes de filiales plus exportations sur C.A. total

(b) ventes des filiales moins importations intra-firmes sur C.A. total

Source : J.H. DUNNING et R.-D. PEARCE "The World's largest industrial enterprises" Gower, Guildford, 1981, pp. 119 et 122.

Tableau 4. Exportations vers les filiales depuis la maison mère (\%)

\begin{tabular}{|c|c|c|c|c|c|c|}
\hline & \multicolumn{3}{|c|}{ Japon } & \multicolumn{3}{|c|}{ Etats-Unis } \\
\hline & 1973 & 1978 & 1983 & 1966 & 1977 & 1982 \\
\hline $\begin{array}{l}\text { Industries manufacturières } \\
\text { dont }\end{array}$ & 24,3 & 26,2 & 29,8 & 36,1 & 37,3 & 41,9 \\
\hline $\begin{array}{l}\text { Industries électriques et } \\
\text { électroniques }\end{array}$ & 35,4 & 48,8 & 24,8 & $\begin{array}{l}28,3 \\
\text { (a) }\end{array}$ & $\begin{array}{l}30,9 \\
\text { (a) }\end{array}$ & $\begin{array}{l}35,1 \\
(\text { a }) \\
(50,4)\end{array}$ \\
\hline
\end{tabular}

(a) Hormis l'informatique sauf chiffres entre parenthèses.

Sources : GERDIC d'après M.I.T.I., op.cit., 1986, p. 96, U.S. Depart. of Commerce, "US Direct Investment abroad : 1982 Benchmark Survey Data" U.S. G.P.O., Washington 1985, 370 pages plus annexes et "U.S. Multinational Companies" U.S. G.P.O. Washington 1983, 84 pages. 
La base productive des industries japonaises est donc comme repliée sur son territoire malgré une première phase de croissance de l'I.D.E., entre 1971 et 1980 . Il nous reste à examiner si le mouvement récent de délocalisation du capital japonais a permis d'approfondir la mondialisation de cette base.

\section{Section II. Le monde contraignant}

A l'orée des années 80 , les opérations sont encore surtout réalisées à partir du territoire national qui constitue presque à lui seul la base productive japonaise. Les frictions, le protectionnisme, le souci de la sécurité des approvisionnements en temps de crise, la conscience des accords nécessaires pour cela ont déjà influé sur les réseaux des grandes entreprises japonaises; le durcissement de la compétition à travers les crises pétrolières devrait les amener à réorganiser une partie de leurs processus productifs couvrant plusieurs parties du monde.

Il est vrai que de nouvelles contraintes se sont accumulées, exigeant un nouveau déploiement des outils de production des Japonais.

\section{$\$ 1$ - Des contraintes insoutenables}

La deuxième étape de croissance de l'investissment japonais à l'étranger commence en 1981. Certaines causes exogènes expliquent ce nouvel élan, mais s'appliquent aussi bien au Japon qu'à d'autres pays industrialisés. Nous citerons, par exemple, les difficultés croissantes de certains pays en voie de développement à drainer des capitaux en provenance de banques commerciales. Situation qui les a amenés à composer davantage avec les intérêts de firmes étrangères pouvant implanter des filiales sur leurs territoires. Nous n'examinerons pas plus avant ces déterminants ${ }^{11}$. En revanche, il existe 
Graphique 1.La substitution entre l'exportation et la production locale par les producteurs japonais de téléviseurs couleur

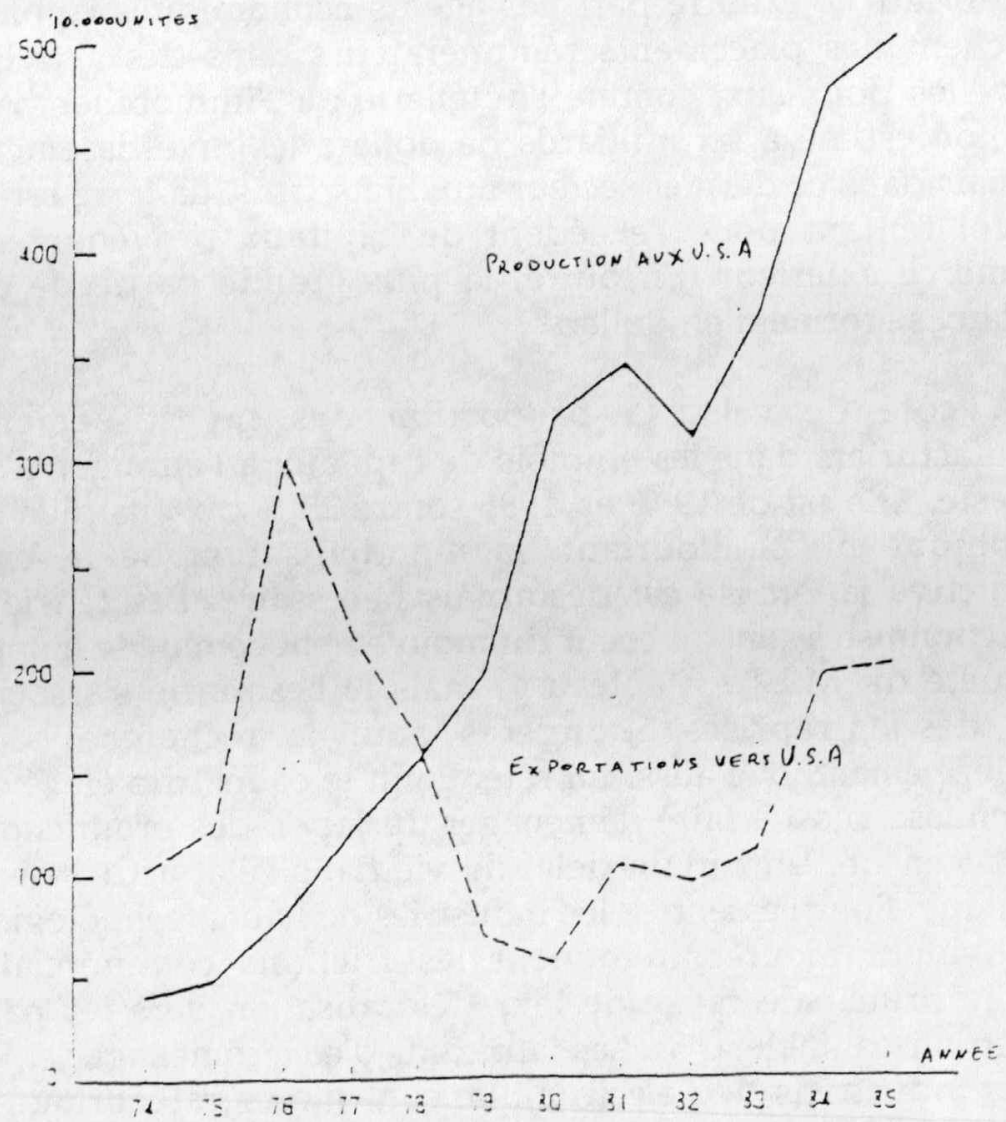

Source : M.I.T.I. "White Paper on International Trade" Tokyo, 1986. 154 pages, p. 74. 
plusieurs explications endogènes au changement de tendance de la croissance de l'I.D.E. japonais. Le graphique 2 montre la forte proportion représentée par les activités non manufacturières. Elles atteignent, entre 1981 et 1984, 72,8\% de l'I.D.E. total, soit $8 \%$ de plus que pendant la période antérieure (cf. tableau 1). D'autre part, les agents économiques japonais cherchent des placements rémunérateurs dans des activités nouvelles pour eux, comme l'hôtellerie ou l'immobilier ${ }^{12}$. En 1986, on estime à six milliards de dollars les investissements japonais dans ce dernier secteur aux Etats-Unis. La terre est un emploi tentant pour l'excédent de capitaux provenant du commerce extérieur. En outre, la plus grande partie de ces capitaux se forment en dollars.

A côté de cela, la proportion des investissements manufacturiers dans les emplois de capitaux à l'étranger reste modeste. Elle est de $19 \%$ en 1985 contre $28 \%$ dix ans plus tôt (graphique $n^{\circ} 2$ ). Pourtant, la mondialisation de la base productive japonaise est désormais nécessaire. Les tensions protectionnistes sont au coeur du mouvement comme le montre l'enquête du M.I.T.I. (tableau 4) mais le besoin de s'associer avec des entreprises étrangères pour la recherche et le développement joue aussi un rôle. Enfin le cours trop élevé du yen pousse aussi à faire déménager du Japon des productions qui ont encore un certain délai de vie dans l'état actuel de la technique. Pour l'essentiel, les industries de haute technologie à forte valeur ajoutée invoquent les frictions commerciales comme principale cause de leur délocalisation vers les pays industrialisés (tableau 5). Les fabricants d'électroménager et les autres industriels de l'électronique sont moins catégoriques : $30 \%$ d'entre eux se fondent sur le protectionnisme. Une proportion plus faible ( $11 \%$ ) fait entrer en ligne de compte le souci de s'adapter à la clientèle. Ainsi, les fournisseurs japonais envisagent de se déplacer en même temps que leurs donneurs d'ordre et clients. 
Graphique 2. Les flux annuels d'I.D.E. du Japon (millions de dollars)

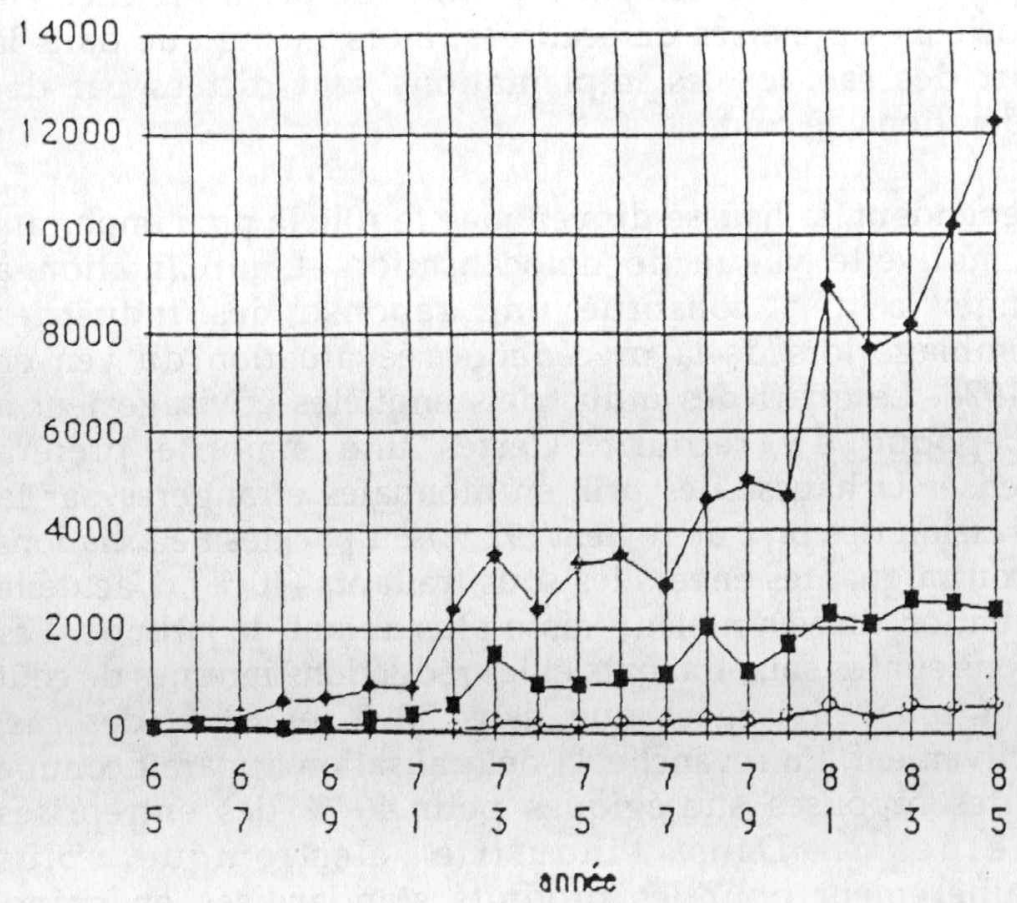

$$
\begin{aligned}
& \text { - Total } \\
& \text { * IEE } \\
& \text { - Manufacturier }
\end{aligned}
$$

Source : GERDIC d'après les données de MOF et du JETRO 
On trouve la même motivation lors des implantations dans les pays en voie de développement. Dans $27,9 \%$ des cas, les entreprises sont motivées par la crainte de perdre la clientèle des sociétés japonaises délocalisées, alors même que dans la plupart des cas, ici, les implantations sont dictées par des considérations de coût.

Cependant, la hausse du yen joue le rôle le plus important de la nouvelle vague de délocalisation. L'implantation à l'étranger a déjà constitué une réponse des industries d'assemblage lors de la précédente réévaluation du yen en 1977-1978. Le quart des industriels enquêtés envisageaient à cette époque d'y recourir. Certes une majorité préféra compenser la hausse des prix en monnaies étrangères par la compression des prix de revient (92\%) ou par des négociations plus contraignantes envers les sous-traitants $(40 \%)$. L'actuelle réévaluation du yen ne leur laisse plus autant de latitude. Les pressions sur les sous-traitants et les réductions internes de coût ne sont plus envisagées que dans $30 \%$ et $80 \%$ des cas, respectivement. En revanche, la délocalisation apparaît comme l'une des réponses stratégiques pour $40 \%$ des entreprises enquêtées ${ }^{13}$. Dans l'industrie électronique, plus particulièrement pour les produits standardisés en grand volume (imprimantes, claviers, moniteurs, E.G.P....), les coûts de transport redeviennent significatifs; ainsi OKI America Inc. a décidé de recourir à la conteneurisation plutôt que le vrac pour transporter ses imprimantes par bateau depuis l'Asie ${ }^{14}$. En revanche, les gains de productivité s'essouflent. En effet, le haut degré d'automatisation déjà atteint par ces industries ne laisse souvent plus le choix que de solutions radicales (risquées, si le marché se modifie) - ou de la délocalisation entraînant avec elle la productivité déjà obtenue. La délocalisation se recommande d'ailleurs non seulement pour compenser la hausse du yen, mais aussi pour réduire les tensions protectionnistes. Or, les causes mécaniques de tension se sont encore aggravées puisque la 
Tableau 5. Les motifs des mouvements de délocalisation des entreprises japonaises

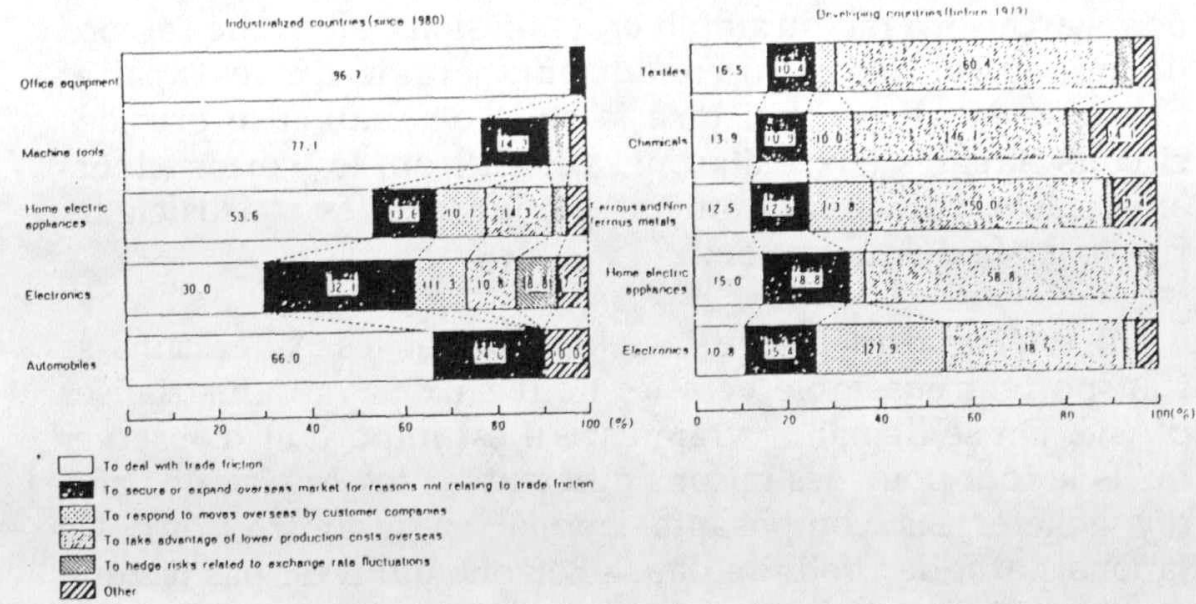

Note : Companies were asked to rate motives for seeking production bases overseas, assuming 100 as their total motives. Results were then totalled by region and industry.

Source : MITI "Questionnaires on the Overseas Production of Japanese Companies" 
parité dollar-yen est tombée à 140 en avril 1987, alors que déjà en 1986 les exportations japonaises d'électronique grand public avaient chuté de $25 \%$ par rapport à l'année précédente.

L'intention de Washington d'imposer une surtaxe de $100 \%$ comme pénalité pour un certain montant de vente de produits finis japonais incorporant des semi-conducteurs acquis à trop bon marché, n'a fait qu'amplifier les tensions, alors que l'accord de juillet sur les semi-conducteurs aurait dû les apaiser durablement. Il est vrai qu'à la suite de celui-ci le prix des circuits intégrés aux Etats-Unis s'éleva trop; les constructeurs américains trouvèrent la même parade que les constructeurs japonais : la délocalisation.

Il est remarquable que les Japonais et les Américains se transportent ensemble vers les nouveaux pays industrialisés d'Asie. On se demande vraiment s'il est important d'observer qu'ils le font pour des raisons différentes : les Américains afin d'y acheter les composants japonais à moindre coût; les Japonais afin de produire dans une zone qui n'est pas la zone yen. Au fond c'est le bénéfice d'une certaine convention non écrite que les uns et les autres cherchent au même endroit; une convention par laquelle il demeurera encore en Asie des zones relativement abritées du protectionnisme.

A l'issue de deux vagues de délocalisation, et alors que la troisième s'intensifie, il nous faut examiner l'état actuel de cette délocalisation afin d'en apprécier l'intensité.

\section{\$2. La délocalisation inégale}

Nous avons vu que l'industrie électronique japonaise est extravertie et reste sur place. Elle compte pour beaucoup dans les exportations ( $22 \%$ du total des exportations japonaises en 1985). Elle est aujourd'hui obligée de changer son organisation 
de production au Japon et à l'extérieur. Une enquête du M.I.T.I. le confirme à propos de la réaction stratégique des entreprises après la forte hausse du yen. $52 \%$ des firmes des I.E.E. ont choisi la délocalisation mondiale, alors que cette proportion n'est que de $15 \%$ pour les industries chimiques, $21 \%$ pour le secteur du matériel de transport ou $8 \%$ pour le textile ${ }^{15}$. D'après un échantillon, nous allons examiner l'ampleur des délocalisations des firmes japonaises, puis leur organisation géographique.

L'industrie électronique japonaise comporte 17.092 établissements territoriaux parmi lesquels 133 ont plus de mille employés et 550 en ont plus de trois cents. L'analyse d'un échantillon composé des 138 premières sociétés japonaises disposant d'une division électronique fait apparaître 940 filiales à l'étrangers en 1984, toutes activités confondues. Parmi ces filiales, 556 sont consolidées alors que $384(41 \%)$ ne le sont pas. L'examen du tableau 6 permet de constater la liaison entre la taille et la délocalisation mondiale des unités de production. Ainsi, les sociétés dont l'effectif dépasse 10.000 salariés ont implanté en moyenne 11 filiales consolidées et 13 filiales non consolidées à l'étranger. En revanche, les sociétés de moins de 5.000 salariés ne disposent, en moyenne, que de 2 filiales consolidées et " $1 / 2$ " filiale non consolidée. La relation est encore plus nette si l'on utilise comme critère le chiffre d'affaires en électronique de ces sociétés (tableau 7). Dans ce cas, les onze sociétés qui réalisent, en 1984 plus de 500 milliards de yens de chiffre d'affaires dans cette activité utilisent, en moyenne, 37 filiales étrangères dont 27 non consolidées, alors que les 79 sociétés dont le chiffre d'affaires en électronique n'atteint pas 50 milliards de yens n'ont que deux établissements (consolidés) à l'étranger. Toutefois, malgré l'imprécision des résultats et l'absence de sociétés de petite taille, nous notons que toutes les classes d'effectifs ou de chiffre d'affaires sont représentées à l'étranger. Sans véritablement confirmer l'ampleur du 
mouvement des sous-traitants de premier ordre, ces résultats les laissent apparaître et viennent étayer notre argument initial.

Tableau 6. Classement des firmes japonaises de l'électronique selon le critère de l'effectif et des filiales (mars 1985)

\begin{tabular}{|c|c|c|c|c|c|c|c|}
\hline \multirow{2}{*}{\multicolumn{3}{|c|}{$\begin{array}{l}\text { Classe } \\
\text { d'effectif }\end{array}$}} & \multirow{2}{*}{$\begin{array}{l}\text { Effectif total } \\
\text { de la classe }\end{array}$} & \multicolumn{3}{|c|}{ Nombre de filiales } & $\begin{array}{l}\text { nombre de } \\
\text { sociétés }\end{array}$ \\
\hline & & & & & & & \\
\hline & $\mathrm{N} \geq$ & 100.000 & 297.765 & 12 & 180 & 192 & 2 \\
\hline 100.000 & $>\mathrm{N} \geq$ & 10.000 & 650.829 & 265 & 140 & 405 & 234 \\
\hline 10.000 & $>\mathrm{N} \geq$ & 5.000 & 86.191 & 52 & 15 & 67 & 127 \\
\hline 5.000 & $>\mathrm{N} \geq$ & 1.000 & 177.449 & 205 & 49 & 254 & 75 \\
\hline 1.000 & $>\mathrm{N} \geq$ & 200 & 18.639 & 22 & 0 & 22 & 26 \\
\hline
\end{tabular}

Source : GERDIC, fichier "JAPCONSO" 
Tableau 7. Classement des firmes de l'industrie électronique Japonaise selon le critère du C.A. Electronique et des filiales étrangères (mars 1985)

\begin{tabular}{|c|c|c|c|c|c|}
\hline \multirow{2}{*}{$\begin{array}{l}\text { Classe de C.A. } \\
\text { électronique } \\
\text { (milliards de yens) }\end{array}$} & \multirow[t]{2}{*}{ C.A. êl. } & Nom & de filiales & & \multirow{2}{*}{$\begin{array}{l}\text { nombre de } \\
\text { sociétés }\end{array}$} \\
\hline & & consolidées & $\begin{array}{l}\text { mon } \\
\text { consolidées }\end{array}$ & total & \\
\hline
\end{tabular}

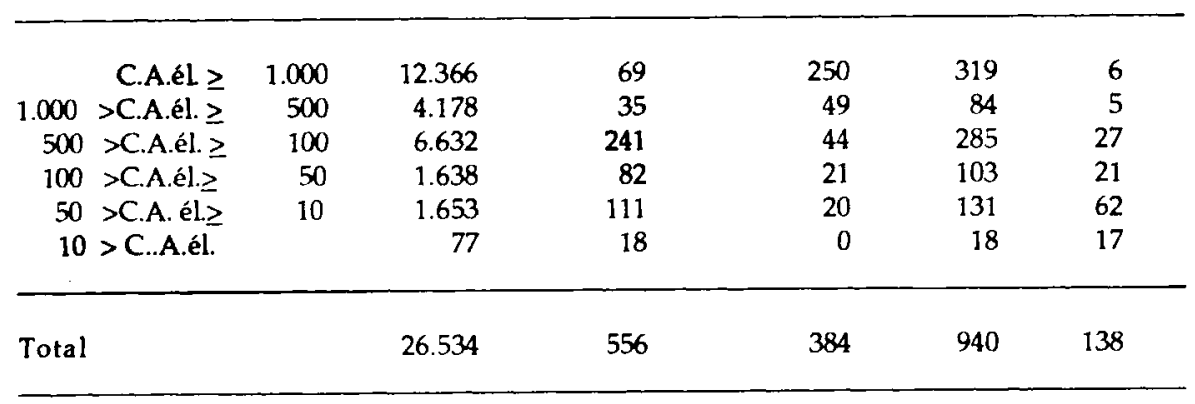

Source : GERDIC, fichier "JAPCONSO".

Si nous examinons les résultats publiés par l'Electronic Industries Association of Japan, selon lesquels, en 1985, les sociétés japonaises auraient 348 établissements à l'étranger (tableau 8), l'Asie du Sud-Est et la Corée constituent le lieu privilégié de ces implantations. Ainsi, 57,4 \% des filiales comptabilisées se trouvent en ASEAN et dans les N.P.I. d'Asie contre $9,4 \%$ en Amérique Latine. Bien entendu, ces établissements sont affectés aux tâches les moins mécanisables et il n'est pas étonnant qu'en ASEAN, 30,5\% des unités de production produisent des biens de consommation et $63 \%$ des composants électroniques. En Amérique du Nord, bien que la structure par activité des établissements soit loin d'être stabilisée, la proportion d'établissements japonais qui produisent des composants est de $40,7 \%$ contre $31,4 \%$ pour 
l'électronique grand public, mais $27,9 \%$ pour les biens d'équipement industriel. Dans les N.P.I., la proportion d'établissements produisant des équipements industriels atteint tout juste $5 \%$. Dans l'état actuel des choses, les implantations étrangères japonaises sont donc le fait des grands groupes. Elles concernent surtout les pays où la main-d'oeuvre est peu onéreuse, et les activités qui comportent des produits très standardisés ou bien dans lesquelles la proportion de travail ouvré reste importante. Ces implantations ont le caractère qu'on a identifié pour les délocalisations de la fin des années 60 et du début des années 70 . On notera tout de même la prépondérance de trois pays (Taiwan, République de Corée et Singapour) avec $42 \%$ du total des filiales.

Le bilan qui vient d'être effectué a permis d'apprécier le caractère doublement inégal des délocalisations de l'industrie électronique japonaise. Inégales surtout parce qu'elles se trouvent concentrées en peu d'endroits. Une remarque supplémentaire doit encore être faite : un très grand nombre d'unités de production satellite fabriquant des composants passifs ou des pièces de radio y figurent (142 établissements au total). Il se peut que ces établissements aient été attirés seulement par le moindre coût de la main-d'oeuvre, mais cela n'expliquerait pas la concentration (à moins qu'on ne tienne compte du fait que Taiwan et la Corée parlaient japonais il y a 40 ans). Il faut chercher de meilleures explications et la corrélation avec les pays d'implantation des fabricants de produits grand public est assez nette pour fonder l'hypothèse de délocalisation interactive, c'est-à-dire provoquée par ces fabricants.

Ces remarques doivent être nuancées puisque, comme pour l'ensemble de l'industrie manufactuière japonaise, les établissements à l'étranger ne représentent encore, dans le cas des industries électroniques japonaises, qu'une faible 
proportion de l'ensemble des établissements. Ainsi, en 1982, seule année pour laquelle nous disposions d'une ventilation des établissements par activité sur le territoire et à l'étranger, les unités de production à l'étranger ne représentent que $2,4 \%$ du total (tableau 8). Cette proportion tombe à $1,4 \%$ dans le cas de l'électronique professionnelle. La segmentation territoriale de la production électronique japonaise apparaît donc à peine entamée. La base productive territoriale reste largement prédominante.

Tableau 8. Nombre d'unités de production des industries électroniques japonaise à l'étranger (1985)

\begin{tabular}{|c|c|c|c|c|c|c|}
\hline & \multirow[b]{2}{*}{$\begin{array}{l}\text { Consumer } \\
\text { Electronic } \\
\text { Equipment }\end{array}$} & \multirow[b]{2}{*}{$\begin{array}{l}\text { Industrial } \\
\text { Electronic } \\
\text { Equipment }\end{array}$} & \multicolumn{4}{|c|}{ Electronic components and devices } \\
\hline & & & $\begin{array}{l}\text { Electronic } \\
\text { components }\end{array}$ & $\begin{array}{l}\text { Electronic } \\
\text { active } \\
\text { Device }\end{array}$ & Others & Total \\
\hline U.K. & 10 & 1 & 2 & 1 & 1 & 15 \\
\hline Ireland & & & & 2 & & 2 \\
\hline Netherlands & & 1 & & & & 1 \\
\hline Belgium & 2 & & 2 & & & 4 \\
\hline France & 3 & 1 & & & 1 & 5 \\
\hline F.R. Germany & 7 & 1 & 5 & 2 & 1 & 16 \\
\hline Spain & 4 & & & & & 4 \\
\hline Europe & 26 & 4 & 9 & 5 & 3 & 47 \\
\hline Canada & 3 & & & 1 & 1 & 5 \\
\hline U.S.A. & 14 & 15 & 13 & 5 & 2 & 49 \\
\hline North America & 17 & 15 & 13 & 6 & 3 & 54 \\
\hline Republic of Korea & 5 & 4 & 32 & 1 & & 42 \\
\hline Taiwan & 16 & 5 & 40 & 3 & 1 & 65 \\
\hline
\end{tabular}




\begin{tabular}{|c|c|c|c|c|c|c|}
\hline Hong Kong & 8 & 1 & 4 & & 1 & 14 \\
\hline Singapore & 9 & 3 & 21 & 2 & 5 & 40 \\
\hline Malaysia & 14 & & 12 & 1 & & 27 \\
\hline Indonesia & 2 & & 1 & & & 3 \\
\hline Philippine & 4 & & 1 & 1 & & 6 \\
\hline India & 3 & & & & & 3 \\
\hline Australia & 4 & 1 & & & & 5 \\
\hline New Zealand & 1 & 1 & & & & 2 \\
\hline Asia and Oceania & 66 & 15 & 111 & 8 & 7 & 207 \\
\hline Ivory Coast & 1 & & & & & 1 \\
\hline Ghana & 1 & & & & & 1 \\
\hline Nigeria & 1 & & & & & 1 \\
\hline Kenya & 1 & & & & & 1 \\
\hline Tanzania & 1 & & & & & 1 \\
\hline Zimbabwe & 1 & & & & & 1 \\
\hline Iran & 1 & & & & & 1 \\
\hline $\begin{array}{l}\text { Africa and Middle } \\
\text { East }\end{array}$ & 7 & & & & & 7 \\
\hline Mexico & 2 & 1 & 1 & & 2 & 6 \\
\hline Guatemala & 1 & & & & & 1 \\
\hline El Salvador & 1 & & & & & 1 \\
\hline Costa Rica & 1 & & & & & 1 \\
\hline Venezuela & 4 & & & & & 4 \\
\hline Brazil & 9 & 2 & 8 & & & 19 \\
\hline Argentina & 1 & & & & & 1 \\
\hline $\begin{array}{l}\text { Central and South } \\
\text { America }\end{array}$ & 19 & 3 & 9 & & 2 & 33 \\
\hline Total & 135 & 37 & 142 & 19 & 15 & 348 \\
\hline
\end{tabular}

Source : Electronic Industries Association of Japan "Facts and Figures on the Japanese Electronics Industry", Tokyo, 1986, p. 13. 
Tableau 9. Le nombre d'unités de production japonaises en électronique selon le critere d'implantation (12/1982)

\begin{tabular}{|c|c|c|c|}
\hline & \multicolumn{2}{|c|}{ Nombre d'établissements } & total \\
\hline $\begin{array}{l}\text { biens d'équipement } \\
\text { composants }\end{array}$ & $3.710(24 \%)$ & $54(14 \%)$ & 3.764 \\
\hline $\begin{array}{l}\text { composants } \\
\text { dont }\end{array}$ & $6.798(44 \%)$ & & \\
\hline $\begin{array}{l}\text { tubes et semi-conducteurs } \\
\text { biens de consommation }\end{array}$ & $\begin{array}{r}449(3 \%) \\
4996(32 \%)\end{array}$ & $129(33 \%)$ & 5.125 \\
\hline total électronique & $15.502(100 \%)$ & $386(100 \%)$ & 15.000 \\
\hline total indust. manufact. & 427.870 & env. 10.000 & env. 438.000 \\
\hline
\end{tabular}

§3. Une nouvelle polarisation (?)

Pour tirer un meilleur parti de son avantage technologique, la base productive japonaise est donc amenée à accentuer sa mondialisation. Si la réévaluation constante du yen constitue un argument en faveur de la délocalisation vers les zones où la main-d'oeuvre est peu onéreuse, ce sont avant tout les tensions protectionnistes qui motivent les industriels japonais. Après la convention nippo-américaine de 1976 concernant les téléviseurs, les autorités européennes dressèrent également des barrières. Puis, en 1982, les Européens s'efforcèrent de limiter les importations de magnétoscopes. Enfin, les semi-conducteurs figurent désormais parmi les produits soumis à une législation douanière aux Etats-Unis et sont taxés en douane en Europe. C'est donc plus particulièrement dans ces deux zones que l'on s'attend à voir le plus de modifications dans la disposition géographique des unités de production nippones ${ }^{16}$. 
En janvier 1986, il existe 201 filiales japonaises en Europe, contre 118 un an plus tôt. Avec 47 établissements en 1985, l'industrie électronique pèse un poids non négligeable dans ce total. Les projets de coopération entre firmes japonaises et européennes seront multipliés. Les appels du pied de la plupart des Etats de la C.E.E. et, plus spécifiquement, des régions touchées par l'effondrement des industries lourdes, ont rejoint la volonté de délocalisation des industriels nippons. En septembre 1986, il y avait une cinquantaine de filiales pour l'électronique, âgées de moins de 10 ans - de moins de 6 dans la plupart des cas - consacrées à l'électronique grand public dans la proportion de $70 \%$ ( $16 \%$ seulement aux composants). Les perspectives d'implantation n'ont pas remis en cause l'avance de l'électronique grand public jusqu'à présent : les plus récents projets sont orientés vers la fabrication de magnétoscopes ou de lecteurs de disques compacts (tableau 10). 
Tableau 10. Les délocalisations projetées vers l'Europe

\begin{tabular}{|c|c|c|c|c|}
\hline Soctéte & Produits & $\begin{array}{l}\text { Pays } \\
\text { destinatalre }\end{array}$ & $\begin{array}{l}\text { Nature et montant } \\
\text { of } 1,1 \text { nvestissement }\end{array}$ & $\begin{array}{l}\text { Date de mise en } \\
\text { service prevue }\end{array}$ \\
\hline $\begin{array}{l}\text { Matsushita } \\
\text { Electranic Industries }\end{array}$ & $\begin{array}{l}\text { Composants } \\
\text { Mécanismes de } \\
\text { magnétoscopes VHS }\end{array}$ & R.F.A. & 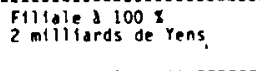 & $\begin{array}{l}\text { Pendant loannese } \\
\text { fiscale } 1986\end{array}$ \\
\hline $\begin{array}{l}\text { Matsusnita } \\
\text { Electronic Induseries }\end{array}$ & $\begin{array}{l}\text { Composants } \\
\text { electroniques }\end{array}$ & R.F.A. & $\begin{array}{l}\text { Joint venture a } \\
50 \text { avec Matsusnita } \\
\text { Electronics Components } \\
\text { l milliford de Yens }\end{array}$ & fin. 1986 \\
\hline Toyo Radiator & $\begin{array}{l}\text { Radiateurs } \\
\text { d'automobilies }\end{array}$ & Europe & $\begin{array}{l}\text { Joint venture } \\
\text { sounaltée }\end{array}$ & Pas encore dectaée \\
\hline Tosniba & Copleurs & France & $\begin{array}{l}\text { Filiale a loo } 5 \\
\text { a millíaras de Yens }\end{array}$ & $\begin{array}{l}100000 \text { unités en } 1987 \\
200000 \text { unités du bout } \\
\text { de } 3 \text { ans }\end{array}$ \\
\hline Tosniod & Magnetoscopes & R.F.A. & $\begin{array}{l}4 \text { milliards de rens } \\
\text { sur } 3 \text { ans }\end{array}$ & $\begin{array}{l}\text { Mars } 1987 \\
120000 \text { unites en } 1987 \\
400000 \text { par an } \\
\text { opres } 3 \text { dns }\end{array}$ \\
\hline Tokyo Electric & Machines a ecrire & A.F.A. & $\begin{array}{l}\text { Filidale } 100 \% \\
1,5 \text { milliaras de Yens }\end{array}$ & $\begin{array}{l}\text { entre l } 000 \text { et } \\
\text { i } 500 \text { unités par mols } \\
\text { d partir de fin } 1986\end{array}$ \\
\hline Sony & DisC CD & Autriche & 6 millilaros de Yens & $\begin{array}{l}\text { Un million de discs } \\
\text { par an d partir de } \\
\text { pété } 1989\end{array}$ \\
\hline Sony & $\begin{array}{l}\text { Apparells CO et } \\
\text { magnétoscopes }\end{array}$ & France & $\begin{array}{l}\text { Fillale a } 100 \\
\text { Plusteurs dizaines } \\
\text { millions de FF }\end{array}$ & $\begin{array}{l}10 \text { o00 apparells CD } \\
\text { par an et } 5000 \\
\text { magnétoscope } 3 \mathrm{~mm} \\
\text { arant fin } 1986\end{array}$ \\
\hline $\begin{array}{l}\text { Ploneer } \\
\text { Electrontc }\end{array}$ & $\begin{array}{l}\text { Ampl fficateurs } \\
\text { HI Fi }\end{array}$ & Espagne & $\begin{array}{l}\text { Jofnt venture d } 808 \\
150 \text { millions de Yens }\end{array}$ & julllet 1986 \\
\hline $\begin{array}{l}\text { Yamanouchl } \\
\text { pharmaceutical }\end{array}$ & $\begin{array}{l}\text { Medicaments pour } \\
\text { ulcéres peot ques }\end{array}$ & Irlande & a milifards de Yens & 1988 \\
\hline T.O.K. & Cassettes audio & R.F.A. & $\begin{array}{l}\text { Achat de Grunding Werke } \\
\text { Dlusieurs centaines de } \\
\text { millions de rens }\end{array}$ & 1988 \\
\hline
\end{tabular}

Source : Japon Economie, n² 201, janvier 1987, p. 17. 
Aux Etats-Unis, les statistiques n'offrent pas la même précision qu'en Europe pour les années récentes. Malgré tout, la compilation des annuaires pour l'International Trade Administration (I.T.A.) permet de suivre la proportion de transactions (créations, extensions, fusions, acquisitions) effectuées par les sociétés étrangères en Amérique dans les différentes secteurs industriels. Le GERDIC a ainsi identifié 437 opérations industrielles effectuées par des firmes étrangères dans le domaine de l'électronique entre 1974 et 1984. La part du Japon dans ces activités atteint presque $30 \%$ avec 129 transactions recensées, mais l'année charnière se situe en 1978, date à laquelle leur nombre sextuple, passant de 3 à 17 . Le mouvement s'amplifie à nouveau en 1984 , avec 29 transactions contre 17 en 1982. Il est encore chaotique, comme en témoigne le graphique $n^{\circ} 3$, mais le recul relatif des industriels européens et canadiens semble se dessiner au profit du Japon et des autres pays, ici ceux d'Asie.

Il s'agit bien dans ce cas de "tariff factories". D'ailleurs, comme en Europe, la plus large part des transactions concernent la création de nouvelles unités de production $(45 \%)$ ou bien l'acquisition et l'extension d'établissements existants (50 $\%$ ), il ne reste que $5 \%$ pour les filiales communes.

Comme toujours, les maisons de commerce sont les principaux créateurs japonais de filiales. Les banques d'investissement (Securities Companies) s'y sont jointes récemment à la faveur de l'établissement de capital ventures en Amérique de l'ouest - leurs objectifs allient la plus value technique à la plus value réclamée par leurs commettants (plus value technique au profit des sociétés manufacturières de leur clientèle). La plupart des nouvelles entreprises électroniques japonaises commencent par la production OEM (fournitures d'appareils que des constructeurs américains incorporent dans leurs systèmes en les rebaptisant); elles savent produire en 
nombre des marchandises de bonne qualité pour les besoins de la chaîne (just in time). Leur savoir-faire est industriel : elles ont rarement une technologie nouvelle à développer. En 1985, neuf cent d'entre elles ont des observatoires sur la Côte Ouest et, chaque mois, une dizaine d'autres viennent encore chercher des partenaires éventuels pour une coopération.

Graphique 3. Les transactions étrangères dans l'électronique aux EtatsUnis par zone d'origine des capitaux

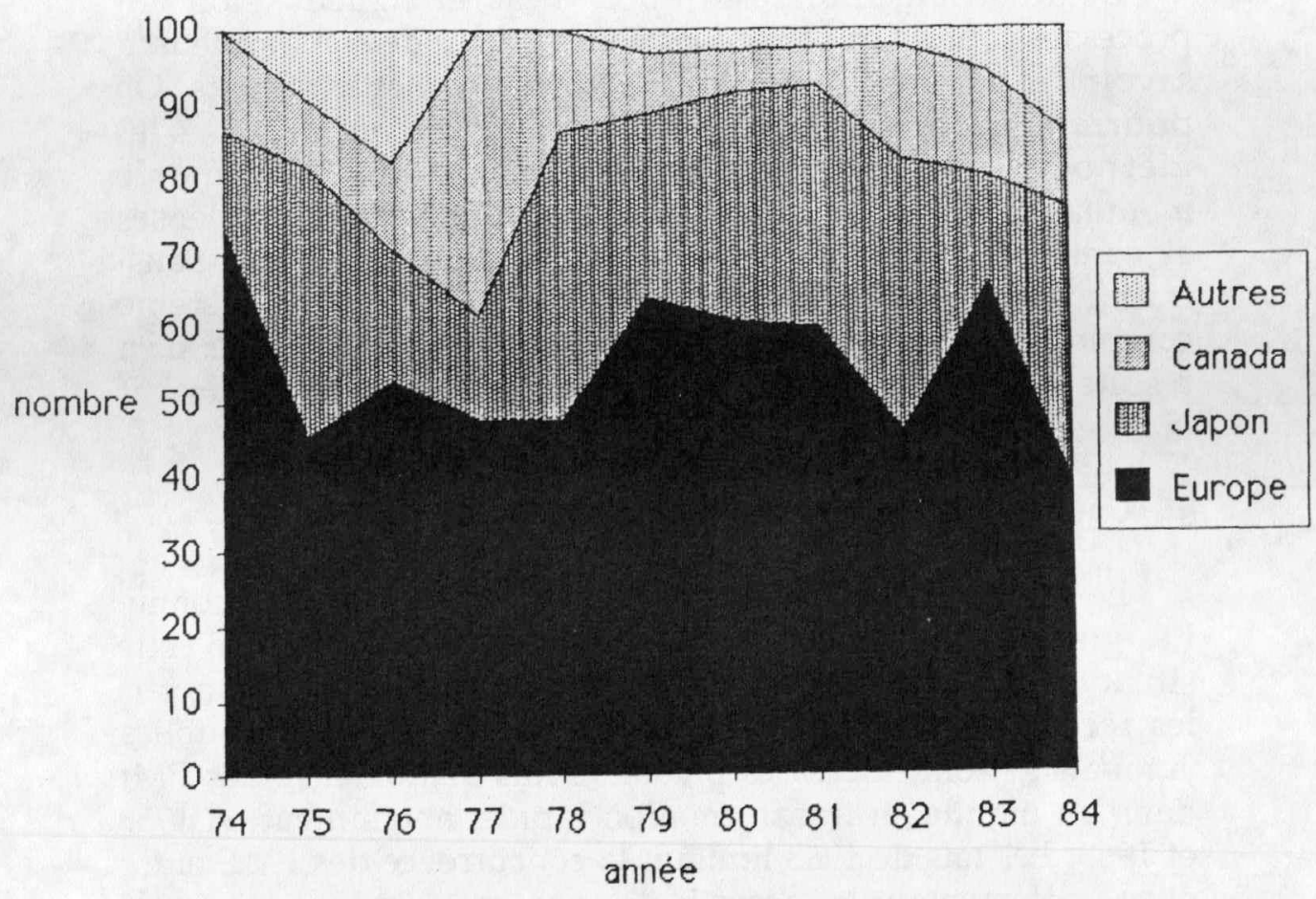

Source : GERDIC 
L'industrie électronique japonaise qui avait initialement privilégié l'hinterland du Pacifique a été récemment amenée à porter sa base productive au-delà de la zone Asie-Pacifique, en particulier à cause des pressions des pays occidentaux. Malgré tout, il y a encore des points obscurs dans cette évolution; pourquoi s'est-elle produite tardivement alors que les pressions extérieures ont été vives et répétées ?

\section{Section III. La systemofacture territoriale}

L'industrialisation du Japon s'est effectuée selon un processus tout à fait particulier compte tenu de son ampleur, à savoir l'expansion d'un tissu très dense de sous-traitants. On pourrait croire ce tissu passif; or, lorsqu'on observe les méthodes de gestion japonaises dans l'industrie manufacturière, on constate l'existence d'inter-relations denses et constantes entre certains donneurs d'ordres et les soustraitants (§1). La principale caractéristique de ce système complexe est sa soumission à des contraintes de proximité. Il en résulte que son transfert d'une région à une autre est délicat (§2).

\section{\$1 - Une hiérarchie complexe et élaborée}

Le caractère dualiste de l'industrie japonaise est bien connu. Les grandes entreprise tirent de nombreux avantages de cette situation qui leur permet de répartir leurs risques et d'amortir les récessions conjoncturelles. Toutefois, les pouvoirs publics japonais se sont intéressés plusieurs fois à la défense des PMI dans un monde en transformation rapide, notamment en 1956 et 1963. Les lois de 1963 limitant la concurrence des PME entre elles, réglementant les accords de sous-traitance, ont consolidé la base productive japonaise, car s'il est vrai que les petites entreprises supportent une large partie des coûts d'ajustement de la production à la demande, il n'en demeure pas moins que 
les liens entre les sous-traitants, et, par extension, les PME d'un côté et les grands groupes de l'autre, sont denses et mutuellement profitables.

Les entreprises sous-traitantes sont très nombreuses au Japon. On en estime le nombre, toutes activités confondues (y compris les services) à 6,2 millions en 1982 contre 3,5 millions en 1960. Leur place dans l'industrie japonaise est énorme. Le tableau 11 permet de comparer la structure des industries manufacturières de plusieurs pays. Les firmes occupant 20 à 99 salariés représentent $85 \%$ de l'ensemble des entreprises japonaises de plus de 20 personnes, contre $69 \%$ aux Etats-Unis et $76 \%$ en France. Quant aux entreprises de moins de 20 personnes, elles sont trois fois plus nombreuses au Japon qu'aux Etats-Unis. Du tissu industriel japonais, on peut donc dire qu'il est très dense et composé d'une large proportion de PME dont l'essentiel effectue de la sous-traitance.

La structure attendue se rencontre dans les industries électroniques (tableau 12) d'une façon un peu moins appuyée qu'ailleurs. Toutefois, les sous-traitants sont en général hiérarchisés en trois niveaux, selon une formule un peu identique aux contractants du Department of Defense aux Etats-Unis. Au troisième niveau, nous retrouvons la très petite entreprise, souvent familiale, utilisant des travailleurs temporaires. Au second, et surtout au premier niveau interviennent des firmes qui entrent en rapport direct avec le donneur d'ordres ${ }^{17}$. Les sous-traitants de premier niveau effectuent souvent une sous-traitance de spécialité, c'est-à-dire qu'ils permettent au donneur d'ordres d'éviter d'investir pour atteindre le même degré de spécialité ou de productivité qu'euxmêmes. En revanche, les sous-traitants de troisième niveau organisent quelquefois la sous-traitance de capacité, lorsque le donneur d'ordres ne souhaite pas ajuster instantanément sa production de pièces à ses besoins avec ses moyens propres; le 
plus souvent, ce que le système offre est simplement une soustraitance d'économie, reposant sur des coûts peu élevés de la main-d'oeuvre en général ${ }^{18}$.

Tableau 11. Structure de l'industrie manufacturière dans les grands pays industrialisés selon le critère de l'effectif (nombre d'établissements)

\begin{tabular}{|c|c|c|c|c|c|}
\hline Pays d'implantation & $\begin{array}{l}\text { Royaume Uni } \\
\text { (4) }\end{array}$ & $\begin{array}{l}\text { France } \\
\text { (1) }\end{array}$ & $\begin{array}{l}\text { Allemagne } \\
\text { (1) }\end{array}$ & $\begin{array}{c}\text { Japon } \\
\text { (2) }\end{array}$ & $\begin{array}{c}\text { Etats-Unis } \\
\text { (3) }\end{array}$ \\
\hline $\begin{array}{l}\text { Effectifs } \\
\text { employés }\end{array}$ & 1983 & 1982 & 1982 & 1980 & 1981 \\
\hline $\begin{array}{l}1.000 \text { et }+ \\
500 \text { a } 999\end{array}$ & $\begin{array}{r}658 \\
1.120\end{array}$ & $\begin{array}{l}517 \\
878\end{array}$ & $\begin{array}{r}939 \\
1.336\end{array}$ & $\begin{array}{r}647 \\
1.150\end{array}$ & 2.000 \\
\hline 200 à 499 & 3.397 & 3.164 & 4.087 & 4.097 & $\begin{array}{r}13.000 \\
(5)\end{array}$ \\
\hline $\begin{array}{c}100 \text { à } 199 \\
50 \text { à } 99\end{array}$ & $\begin{array}{l}4.732 \\
7.238\end{array}$ & $\begin{array}{l}4.693 \\
7.696\end{array}$ & $\begin{array}{l}5.952 \\
9.561\end{array}$ & $\begin{array}{r}8.131 \\
18.157\end{array}$ & $\begin{array}{r}23.000 \\
(6)\end{array}$ \\
\hline 20 à 49 & 16.000 & 21.177 & 18.306 & 60.947 & 85.000 \\
\hline Total & 33.145 & 38.110 & 40.181 & 93.129 & 123.000 \\
\hline$-\operatorname{de} 20$ & $\begin{array}{l}\text { nd } \\
\text { nd }\end{array}$ & nd & $\begin{array}{r}7.204 \\
(7) \\
47.285\end{array}$ & $\begin{array}{l}641.494 \\
734.623\end{array}$ & 198.000 \\
\hline
\end{tabular}

\section{Sources et notes :}

1. J. NORTHCOTT "Microelectronics in industry : An International Comparison" Policy Studies Institue, $n^{\circ} 635$, p. 57.

2. Office Franco-Japonais "Le Japon en chiffres : 1985", Paris, décembre 1984, p. 37.

3. Bureau of the Census "Statistical Abstract of the United States : 1984", U.S. Department of Commerce, U.S.-G.P.O., décembre 1983, p. 534.

4. Japon Economie, $\mathrm{n}^{\circ} 180$, p. 9.

5. 250 à 999 employés.

6. 100 à 149 employés.

7. CSO "Annual Abstract of Statistics : 1983 "Government Statistical Service, $\mathrm{n}^{\circ} 121$, Londres, 1985, p. 123. 
Tableau 12. Structure de l'industrie Japonaise selon le critère de l'effectif (nombre d'établissements en 1983)

\begin{tabular}{lrrrr}
\hline Effectifs & \multicolumn{2}{l}{ Toutes industries manufacturières } & \multicolumn{2}{c}{ Electronique } \\
\cline { 2 - 5 } & \multicolumn{1}{c}{ n } & $\%$ & $\mathrm{n}$ & $\%$ \\
& & & & \\
\hline & 413.531 & 92,5 & 13.550 & 79,2 \\
4 à 49 & 29.852 & 6,7 & 2.992 & 17,5 \\
50 à 299 & 2.909 & 0,6 & 417 & 2,5 \\
300 à 999 & 650 & 0,2 & 17.092 & 100 \\
1.000 et + & & & & \\
\hline
\end{tabular}

Source : GERDIC d'après les données de l'E.I.A.J. "Facts and Figures", Tokyo, 1986, p. 15.

La place de la sous-traitance dans l'industrie électronique japonaise la fait ranger à part de l'industrie mondiale. Le tableau 13 présente les rapports de la valeur ajoutée au chiffre d'affaires dans trois secteurs de l'électronique aux Etats-Unis. Dans les trois cas, le rapport varie du simple au double en faveur des Etats-Unis. Un tel contraste ne peut pas être dû aux seules méthodes de comptabilisation ${ }^{19}$. A l'évidence, le mode d'organisation de l'industrie japonaise diffère sensiblement de celui de son homologue nord-américain. On observe que malgré leur fort degré d'intégration - bien qu'ils fabriquent eux-mêmes leurs composants actifs - les groupes japonais de l'électronique recourent profondément à la sous- traitance. La coopération entre fournisseurs et donneurs d'ordres est intime. 
Tableau 13. Rapport de la valeur ajoutée au C.A. des industries électroniques : critère national (\%)

Secteur Japon (1984) Etats-Unis (1983)

$\begin{array}{lll}\text { Equipements électron.prof. } & 30,5 & 61,3 \\ \text { Composants électroniques } & 35,9 & 62,7 \\ \text { Electro-ménager et électronique } & 19,6 & 42,6 \\ \text { Grand Public } & & \end{array}$

Total

Source : GERDIC d'après l'E.I.A.J. et l'E.I.A.

L'originalité de l'industrie japonaise est d'avoir développé très loin vers le bas les méthodes de contrôle de qualité et l'approvisionnement au bon moment (JIT = just in time). Ces procédés réduisent considérablement les stocks, mais il faut que les produits intermédiaires soient absolument fiables. Les fournisseurs sont amenés à s'aligner sur les normes de qualité de leurs donneurs d'ordres qui, de leur côté, vont sélectionner un très petit nombre de sous-traitants. Obtenant des commandes plus longues, ceux-ci vont s'efforcer d'amener leurs propres fournisseurs au même comportement. En ce sens, les programmes de qualité sont structurants vers l'amont. Raphaël Kaplinski qualifie le J.I.T. de "relation organique et systémique entre fournisseur et donneur d'ordres"20. Jean-Paul Valla évoque, de son côté, l'existence d'un système fournisseur/client interactif :

"De fait, l'établissement d'une relation profitable avec un fournisseur ou un client industriel représente un investissement tel que cet investissement... explique en 
partie l'étonnante stabilité de ces relations dans le temps"21.

L'existence d'une relation de sous-traitance débouche donc déjà sur une certaine stabilité du système fournisseur client. $\mathrm{Si}$ on lui juxtapose des exigences de qualité et les contraintes du J.I.T., la relation devient infiniment complexe. Le texte évoqué plus haut illustre cette attitude particulière des entreprises japonaises :

"In the Quest to eliminate defects, Japanese firms have concentrated on raising the level of automation, on using superior materials and on implementing more effective control procedures"22.

Dans bien des cas, notamment lorsqu'il y a développement de produits en commun, l'investissement relationnel est tel que des barrières à l'entrée doivent être établies. Jean-Paul Valla parle de système fournisseur client ${ }^{23}$. Cela nous semble fréquemment le cas dans l'électronique japonaise entre le donneur d'ordres et les sous-traitants de premier rang, voire de second rang, avec lesquels nous croyons voir se développer des "économies de système entre firmes" en plus des économies de systèmes internes, propres aux donneurs d'ordres dont les établissements sont très automatisés. Il y a donc existence d'une Systémofacture, au sens de Raphaël Kaplinski ${ }^{24}$, dont il nous semble qu'elle constitue une des causes de l'immobilisme territorial relatif des producteurs japonais.

$\$ 2$ - Un ensemble difficile à désarticuler

L'organisation du travail aux Etats-Unis n'était probablement pas tout à fait ce qu'elle aurait dû être quand les entreprises américaines ont voulu adopter pour elles-mêmes la méthode sans stocks (just in time) et le contrôle de qualité. Leurs 
déboires nous ont aidé à comprendre la fragilité de la systémofacture propre aux firmes japonaises.

I.B.M. a commencé à généraliser ces méthodes en 1980, Control Data (C.D.C.) en 1981, D.E.C. Xerox, General Electric ont suivi. Ce mouvement a engendré une diminution considérable du nombre de fournisseurs, puisque seuls ceux qui répondaient aux nouvelles normes de qualité et de livraison ont été maintenus. Ainsi, chez Xerox, il a été ramené à 5.000 à 300 , chez C.D.C. de 1.100 à 300; chez Apple, il a été réduit de moitié et chez I.B.M. il a diminué de 550 à $250^{25}$. Outre le nombre, le critère de sélection a aussi porté sur la proximité géographique, contrainte issue de la volonté d'obtenir des stocks faibles ou inexistants (close spatial relationship : long terme relationship).

Lorsqu'I.B.M. cherche des fournisseurs à moins d'un jour de route (one day trucking), c'est pour mieux coopérer avec eux et surveiller la qualité. Dieter Ernst avait d'ailleurs fait remarquer que les coûts d'acheminement des pièces détachées étaient trop élevés dans la sous-traitance internationale, entre des segments du processus de production désignés sans qu'on s'inquiète de ces coûts.

Avec l'organisation rationnelle des processus productifs selon des critères d'automatisation et de contrôle de qualité, de nouvelles contraintes apparaissent pour les groupes industriels. Elles se résument en une proximité géographique et technologique des sous-traitants et des donneurs d'ordres. Or l'industrie japonaise et plus particulièrment l'industrie électronique ont installé ces méthodes depuis une vingtaine d'années. Ayant construit des systémofactures, elles ont rencontré lorsqu'elles ont eu à se délocaliser, la dilatation des systèmes fournisseur-clients, voire leur éclatement. Il n'est donc pas étonnant de constater que chaque vague de délocalisation des fabricants de produits finis ait été suivie par celle de 
fabricants de pièces détachées et accessoires, dans l'optique d'une reconstitution d'un système fragilisé. Dans bien des cas, le pouvoir structurant des méthodes de gestion a permis de trouver des fournisseurs locaux, avec l'aide des sous-traitants de premier niveau implantés à proximité. De telle sorte que désormais, la proportion de pièces achetées localement par les filiales japonaises atteint $75 \%$ dans les pays industrialisés, si l'on excepte les établissements affectés à l'assemblage, contre, $49 \%$ dans les pays en voie de développement (tableau 14). 
Tableau 14. Origines des pièces détachées et accessoires des filiales ateliers Japonaises (toutes industries confondues)
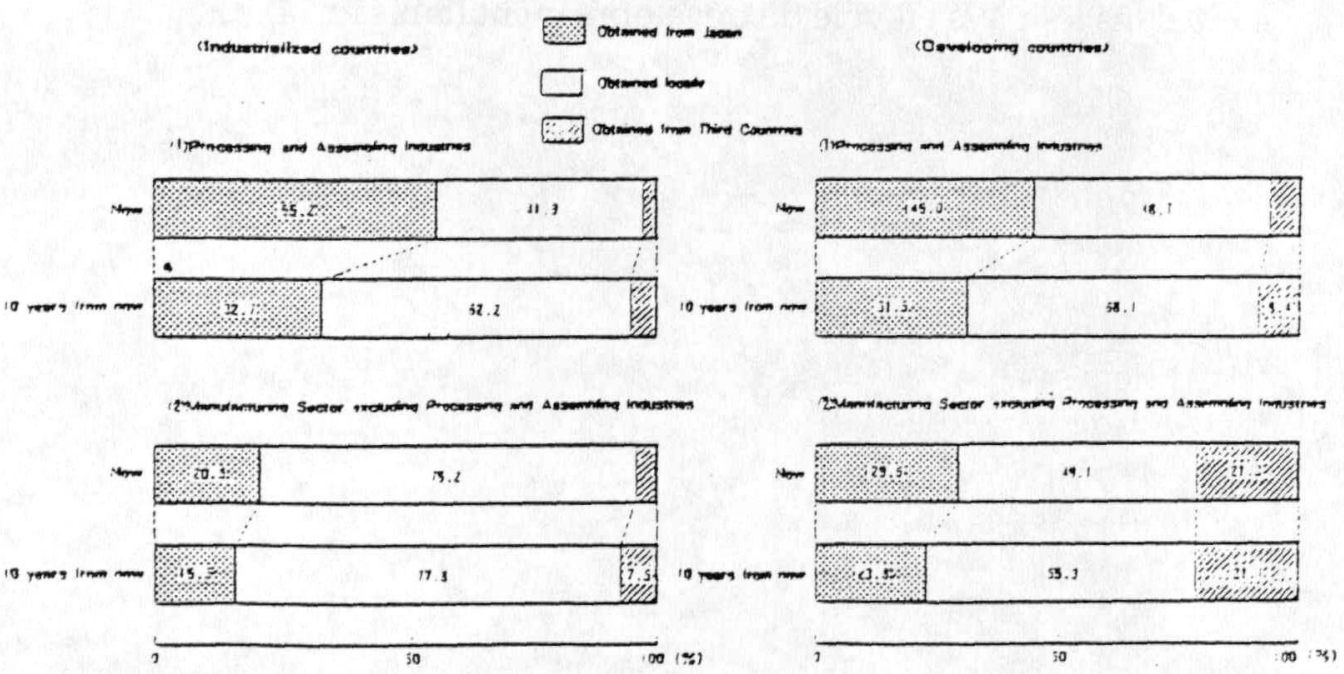

Source : M.I.T.I., op.cit., p. 76. 
Parlant des Multinationales, Charles Albert Michalet a dit comment à son avis elles s'organisent pour atténuer les conséquences des incidents qu'elles ne peuvent pas prévoir: "le principe de base de l'organisation des multinationales : l'internationalisation contre l'incertitude"29. Les groupes japonais ne font sans doute pas autrement, mais leur mode d'internalisation se trouve dans leurs relations avec les soustraitants. Dans ces conditions, les sous-traitants accompagnent les donneurs d'ordre ${ }^{30}$. Dans l'électronique leurs déménagements avaient déjà été vus au début des années $1970^{31}$. C'est pourquoi les sous-traitants en général et plus particulièrement les P.M.E. sont des acteurs importants du mouvement de délocalisation. On estime qu'ils sont responsables de 20 à $30 \%$ des opérations d'I.D.E. ${ }^{32}$.

Il faut toutefois faire place à la leçon que quelques groupes particuliers semblent avoir tiré de leurs expériences. Uniden, un fabricant de matériel de communication qui a implanté toute sa production en Asie, affirme qu'au bout de quelques années la coopération technique permet d'obtenir dans les nouveaux pays industrialisés des produits intermédiaires d'une qualité équivalente à celle des produits japonais ${ }^{33}$. La systémofacture est donc géographiquement transférable parce qu'elle a des effets structurants incontestables et les implantations japonaises en Asie du Sud-Est ont probablement contribué au développement de tissus industriels territoriaux, mais les délais nécessaires à cette reconstitution, à ce remembrement, bien qu'ils ne nous soient pas connus, sont assez longs, et laissent augurer de l'existence d'une "fenêtre de vulnérabilité" pour les groupes japonais qui en tentent l'expérience. 


\section{Conclusion}

L'essor multinational des firmes japonaises de l'électronique est un remède aux surplus commerciaux.

Embryonnaire, pour ne pas dire inexistant, jusqu'au début des années 1970, cet essor a eu pour cause principale la montée du protectionnisme sur les marchés occidentaux. Auparavant, les groupes japonais s'étaient occasionnellement servi des avantages procurés par les faibles salaires de la main-d'oeuvre asiatique. On avait alors constaté que ces délocalisations ne provoquaient pas un éclatement par leur systémofacture, dans la mesure où des sous-traitants accompagnaient le mouvement, et où certains pays d'Asie du Sud-Est offraient un environnement propice à l'industrie ${ }^{34}$.

Les réticences des groupes japonais à se délocaliser tous azimuts, à l'instar des sociétés nord-américaines, semblent tenir à leur mode d'organisation de la production. Désintégrés verticalement, si l'on observe la part de la valeur ajoutée dans le chiffre d'affaires, ils semblent au contraire intégrés si l'on considère qu'ils produisent les composants des appareils euxmêmes. Mais plutôt qu'une "intégration" nous avons découvert une "quasi-intégration", plus délicate à élaborer que la première car reposant sur une interaction constante entre fournisseur et client, entre donneur d'ordres et sous-traitant. De ce fait, si la greffe des groupes industriels japonais à l'étranger s'opère bien, elle devrait contribuer à un apprentissage technologique notable des producteurs locaux, comme cela semble avoir été le cas à Taiwan, à Hong-Kong ou en République de Corée.

Les coûts de la main-d'oeuvre ne suffisent pas à déterminer l'implantation manufacturière et l'approvisionnement mondial. Désormais - probablement depuis déjà un certain temps pour les grands producteurs japonais - d'autres caractéristiques 
doivent être regardées comme déterminantes, notamment la proximité des fournisseurs/sous-traitants de composants et de matériel, les infrastructures industrielles, ainsi que la fiabilité de la qualité des livraisons. Dans le cas des groupes japonais, cette contrainte est d'autant plus drastique qu'ils orientent leur production vers des produits à valeur ajoutée élevée, en général plus complexes. Pour les produits fabriqués en grand volume et à faible coût, leur stratégie n'est pas encore lisible. Les grandes marques japonaises incorporent aujourd'hui des produits finis coréens. Elles y trouvent leur avantage, tout comme les marques américaines ont un avantage à vendre des téléviseurs ou des magnétoscopes japonais. La délocalisation n'est pas une panacée. Pour les marchés que de simples renversements du cours des changes peuvent assécher, ou féconder cycliquement (ou pendant un petit nombre d'années) des solutions plus flexibles que la délocalisation sont souvent recherchées. D'où la réponse inattendue des firmes japonaises qui s'efforcent avant tout de conserver les parts de marché liées à leurs marques.

Une analyse complète des formes d'organisation mondiale des processus productifs par les groupes japonais reste à effectuer ${ }^{35}$. Au-delà du mouvement quantitatif de délocalisation des établissements, la forme des rapports entretenus avec les fournisseurs ou les sous-traitants, selon le degré de complexité des produits et les variables d'environnement territorial, devrait présenter une grande variété. Bien entendu, ce type de division (la segmentation) internationale des processus productifs (B. Lassudrie-Duchene) contribue elle-même à troubler toute lecture des compteurs que sont pour nos les flux douaniers (et surtout les déterminants des variations de ces flux). 


\section{NOTES}

1. "Une nouvelle approche de la spécialisation internationale", Revue $d^{\prime}$ Economie Industrielle, $n^{\circ} 17,3 e$ tr. 1981, pp. 58-72.

2. Voir l'ouvrage "Dynamique de la spécialistion internationale", Economica, Paris, 1979, 176 pages.

3. On trouvera une analyse minutieuse et prospective des échanges internationaux de produits électroniques dans "Groupe de Prospective des Echanges Internationaux "Prospective et échanges internationaux en électronique", Commissariat Général au Plan, Paris, 1987, 310 pages + annexes.

4. B. LASSUDRIE-DUCHENE, J.C. BERTHELEMY et F. BONNEFOY ont offert récemment une interprétation tout à fait originale des soldes commerciaux qui nuance les interprétations habituelles : "l'importation peut être aussi un facteur d'élasticité et exercer une influence positive sur l'emploi des facteurs de production nationaux" dans "L'importation et la production en France", Economie Prospective Internationale, $\mathrm{n}^{\circ} 29,1 \mathrm{er}$ tr. 187, pp. 5-37.

5. "Les multinationales du Tiers Monde dans la mutation du système industriel mondial" Colloque E.A.D.I. W.I.G. "Multinationales" 1987, 18 pages, p. 14.

6. Investissement de développement à l'Etranger.

7. Voir sur ce point A.-J. SCOTT "The semi-conductor Industry in South East Asia : Organization, Location and The International Division of Labour" Régional Studies, vol. 21, n², 1987, 19 p.

8. Office of Technology Assessment, International Competitivness in Electronics, U.S. GPO, Washington, 1983, 546 pages, p. 115.

9. P. DICKEN, The Global Shift : Industrial Change in a Turbulent World, Harper and Row, Londres, 1985, 450 pages, p. 342. 
10. Voir l'article d'A.-J. SCOTT à ce séminaire.

11. Voir par exemple C. OMAN "Changing International Investment in the North South Context", the C.T.C. Reporter, $n^{\circ} 22$, automne 1986, pp. 47-50 ou Center ou Transnational Corporations "Trends and Issues in Foreign Direct Investment and Related Flows", O.N.U., New York, 1985, 96 pages.

12. Tokyo Financial Review, vol. 10, n¹1, novembre 1985, p. 4.

13. M.I.T.I., op.cit ., 1986, p. 68.

14. Electronics Business, novembre 1986, p. 51.

15. Journal of Japanese Trade and Industry, vol. 5, 1986, p. 28.

16. JETRO "White Paper on World and Japanese Overseas Direct Investment", Tokyo, mars 1986, 27 pages.

17. M. YOSHIMORI, "Les entreprises japonaises", P.U.F., Que sais-je ?, Paris, 1984, pp. 97-98.

18. Pour une typologie, voir C. BERTHOMIEU, C. CHARBIT, A. HANAUT et J.T. RAVIT "L'insertion de la France dans la segmentation internationale des processus productifs par la soustraitance internationale", Commissariat Général au Plan, LATAPSES, Nice, 1985, 353 pages + annexes, pp. 30 a 38.

19. Voir sur ce point Electronic Business, 15 avril 1986, pp. 69-76.

20. Dans "Changing Patterns of Industrial Location and International Competition : the Role of TNC's and the Impact of Microelectronics", Bulletin de l'E.A.D.I., $\mathrm{n}^{\circ} 1$, 1985, pp. 69-93.

21. Dans "Elements d'une approche marketing du concept de filières" Revue d'Economie Industrielle, $\mathrm{n}^{\circ} 21,3 \mathrm{e}$ tr. 1982, pp. 76-92.

22. E.I.A.J., op.cit., p. 7.

23. Op.cit ., p. 88. 
24. Op.cit ., p. 72.

25. Electronic Business, 15 janvier 1987, pp. 53-64.

26. M.I.T.I., 1986, p. 77.

27. JETRO, op.cit , p. 22.

28. Journal of Asia Electronics Union, nov.-dec. 1986, p. 43.

29. "Le village planétaire" dans "Une économie mondiale" Pluriel, Hachette, Paris, 1985, pp. 65-105.

30. Journal of Japanese Trade and Industry, vol. 6, n 2 , mars-avril 1987, p. 30.

31. Export-Import Bank, op.cit., 1980, pp. 10-11.

32. Japan Economie, n 191, 28 février 1986, p. 5.

33. Japan Economie, $\mathrm{n}^{\circ} 201$, p. 15.

34. Voir CERNEA "Analyse fine des politiques d'industrialisation dans l'électronique" Commissariat Général au Plan, Paris, 1987, 364 pages.

35. Michael PORTER a recommandé cette étude. Voir M. PORTER, "Competitive Advantage", The Free Press, New York (N.Y.) 1985, pages 414. 


\section{BIBLIOGRAPHIE}

Business Intercommunications Inc. "Directory of Foreign Capital Affiliated Enterprises in Japan", BII, Tokyo, 1985, 799 pages.

Center on Transnational Corporations. "Trends and Issues in Foreign Direct Investment and Related Flows", ONU, New York, 1985, 96 pages.

Commissariat Général du Plan. "L'électronique : un défi planétaire", Prospective des Echanges Internationaux, Paris, décembre 1986, 274 pages.

DEMPA. "Japan Electronics Almanac 1985, Dempa Publications, Tokyo, 1985,882 pages.

P. DICKEN. "The Global Shift : Industrial Change in a Turbulent World", Harper and Row, Londre, 1986, 450 pages.

J. ESMEIN. "Les politiques d'alliance des industriels japonais avec l'étranger". Défense Nationale, août-septembre 1985, pp. 117-133.

The Export-Import Bank. "The internationalization of Japan's Consumer Industry". Research Institute of Overseas Investment, Tokyo, 1980, 25 pages.

JETRO. "White Paper on World and Japanese Overseas Direct Investment". Special Report, Tokyo, 1986, 15 pages.

R. KAPLINSKY. "Changing Patterns of Industrial Location and International Competition : the Role of TNC's and the Impact of Microelectronics". Bulletin de l'EADI, n 1, 1985, pp. 69-93.

MITI. "White Paper on International Trade 1986". Background Information, B1-61, Tokyo, September 1986, 154 pages.

O.T.A. "International Competitiones in Electronics". US Government Printing Office, Washington, 1983, 546 pages.

J.P. VALLA. "Eléments d'une approche marketing du concept de filières", Revue d'Economie Industrielle, $n^{\circ} 21,3$ e tr. 1982, pp. 76-92. 


\section{SUMMARY}

The multinational drive of the Japanese companies in the electronic field is a response to the critics of the growing trade unbalance between Japan and its main partners. However, the Japanese groups remain more reticent to delocalize than the North American firms. This can be explained by their pattern of production organization.

The production process rests on a constant interaction between suppliers subcontractors and clients. As a result, it is more difficult to recreate the same environment abroad. In many cases, main subcontractors accompany the large firms abroad. At the same time, collaboration with local firms should lead to a technological up grading of the latter. It seems that it has already been the case in Taiwan, Hong-Kong and South Korea. 